\section{Adaptive frequency sampling using linear Bayesian vector fitting}

\author{
S. De Ridder ${ }^{\bowtie}$, D. Deschrijver, D. Spina, T. Dhaene and \\ D. Vande Ginste
}

The authors present a novel Bayesian approach to adaptively select frequency samples to obtain a rational macromodel of device responses over a broad frequency range while performing as few electromagnetic simulations as possible. The method leverages a Bayesian approach to vector fitting to construct a data-driven uncertainty measure. The presented technique is demonstrated by application to a double semi-circular patch antenna and is shown to accurately and efficiently construct a rational macromodel over the frequency range of interest.

Introduction: Nowadays, computer-aided design simulations are essential tools in the design phase of modern high speed circuits, due to their increasing complexity, density, and bandwidth. Since linear and passive electromagnetic (EM) systems (such as interconnections, filters, and connectors) are mainly analysed in the frequency domain, adaptive frequency sampling (AFS) schemes are of paramount importance [1-4]. Indeed, simulating such systems through full-wave EM simulators is expensive, given the bandwidth needed in modern applications. AFS schemes allow one to minimise the number of required EM simulations, while simultaneously being able to describe the dynamic behaviour of the system considered in an accurate way [1] A novel macromodelling-based AFS strategy using linear Bayesian vector fitting (LBVF) is proposed in this Letter. It formulates the problem of computing a rational model of the frequency response of the system under study in a Bayesian framework. Numerical results confirm the accuracy and efficiency of the proposed method.

Goal statement: The goal of the AFS is to construct an accurate rational model of the frequency response of the system while performing as few (expensive) EM simulations as possible. Thus, the amount of information obtained by each EM simulation must be maximised. To achieve this, standard AFS techniques compare two or more intermediate models and add, in an ad hoc way, a new frequency sample where they disagree most, in order to reduce uncertainty [1-4]. The novel proposed technique, however, uses the intrinsic uncertainty of the rational models in a Bayesian way.

LBVF framework: The use of Sanathanan-Koerner (SK) iterations for rational macromodelling of device responses has been well established as the vector fitting (VF) method [5, 6]. In this framework, the nonlinear problem of fitting a transfer function $f(s)$ (e.g. S-parameters) with a suitable rational model is linearised by multiplying $f(s)$ with a preliminary denominator $\sigma(s)$

$$
f(s)=\frac{p(s)}{\sigma(s)}=\frac{\sum_{k=1}^{K}\left(r_{k} /\left(s-a_{k}\right)\right)+d}{\sum_{k=1}^{K}\left(\widehat{r_{k}} /\left(s-a_{k}\right)\right)+\widehat{d}},
$$

where $p(s)$ is the numerator and $a_{k}$ are a set of starting poles. The linear system $\sigma(s) f(s)=p(s)$ can now be solved in a least squares sense for the residues $\widehat{r_{k}}$ and $\widehat{d}$ of $\sigma(s)$. Then, the zeros of $\sigma(s)$ can be computed by solving a suitable eigenvalue problem [6]. Since the zeros of $\sigma(s)$ correspond to the relocated poles of $f(s)$, this process can be iterated to convergence by replacing $a_{k}$ with these new poles. Finally, the residues in the partial fraction representation of $f(s)$ can easily be estimated through another linear system.

In the proposed LBVF framework, firstly, a final set of relocated poles is estimated using several iterations of the VF algorithm, as described above. Then, in contrast to traditional SK iteration, samples are drawn from the posterior distribution of $\widehat{r_{k}}$ and $\widehat{d}$, after solving the linearised pole relocation system $\sigma(s) f(s)=p(s)$ using Bayesian linear regression. For each of these samples, the zeros of $\sigma(s)$ and the posterior distribution of the residues of $f(s)$ are calculated using Bayesian multivariate linear regression. Finally, a set of residues is obtained by sampling the corresponding posterior distribution. Each set of the computed poles and residues describes a sample from the posterior distribution of fits to the data. We denote these samples $f_{\mathrm{LBVF}}^{i}(s)$, where $i=1, \ldots, N$ and $N$ is the total number of samples, conditioned on the starting poles. As a result, one LBVF model consists of a distribution of rational models, from which samples can be drawn, while a traditional VF model is formed by a single set of pole/residue pairs.
A prior probability distribution on the residues of $\sigma(s)$ can be specified as a multivariate normal distribution for the residues, times an inverse Wishart distribution for the covariance, in order to yield an analytical solution to the posterior distribution. If no prior information is present, an (uninformative but improper) Jeffreys prior can be used. In that case, the mean of the posterior distribution corresponds to the solution of the classical VF. Analogously, the prior for the residues of $f(s)$ can be specified as a matrix normal distribution times an inverse Wishart distribution. In this Letter, we adopt uninformative priors.

Furthermore, as Bayesian linear regression allows for an analytical form of the marginal likelihood of the data, the pole relocation system can provide a likelihood of the data, conditioned only on the converged poles, and their number. Hence, the novel proposed Bayesian modelling framework offers intrinsic information on the number of poles needed to accurately describe the data. In the standard VF modelling framework, instead, the number of poles is typically chosen ad hoc or through a bottom-up strategy, where the number of poles is iteratively increased until the desired accuracy is reached.

Proposed AFS strategy: Computing the standard deviation of the aforementioned $f_{\mathrm{LBVF}}^{i}(s)$ for $i=1, \ldots, N$ gives a measure of the intrinsic model uncertainty, which naturally lends itself to an adaptive sampling scheme. Since an LBVF model is still conditioned on the number of starting poles (and their location), it is advantageous to consider several LBVF models, with different numbers of starting poles. The importance of each model can be weighted by its marginal likelihood, yielding a data-driven model selection. Hence, a more informative uncertainty measure is the weighted standard deviation of samples from multiple models, with their marginal likelihood as weights. In addition, a small Gaussian penalty is added to avoid choosing frequency points too close to each other.

The proposed adaptive sampling scheme is described in Fig. 1. An initial number of EM simulations is necessary to compute a VF model yielding the relocated poles needed by the LBVF technique, as described before. Hence, only four initial frequency points, uniformly and equidistantly spread over the considered frequency range, are chosen as initial points and LBVF models with different pole numbers are built. Note that it is not possible to use a number of poles higher than the number of frequency points considered. Then, a large number (typically $>500$ ) of samples $f_{\mathrm{LBVF}}^{i}(s)$ is drawn from each model and the corresponding uncertainty measure is calculated. If the uncertainty does not exceed a chosen threshold, the sampling stops and the best model serves as a surrogate for any other frequency. It should be noted that this threshold does not correspond to the fitting accuracy, but to a desired upper limit of the uncertainty measure. The final model is (the mean of) the LBVF model with the highest likelihood. Note that, when using uninformative priors, the mean of this model corresponds to the classical VF solution. If the threshold is surpassed, the frequency point with the maximum uncertainty is chosen and an additional EM simulation is performed for that frequency. The entire process is then iterated until the threshold is no longer exceeded. Since additional frequency points are considered, it is possible to increase the number of poles in each iteration. To curb computation time, only the ten highest order models are retained, while the others are discarded.

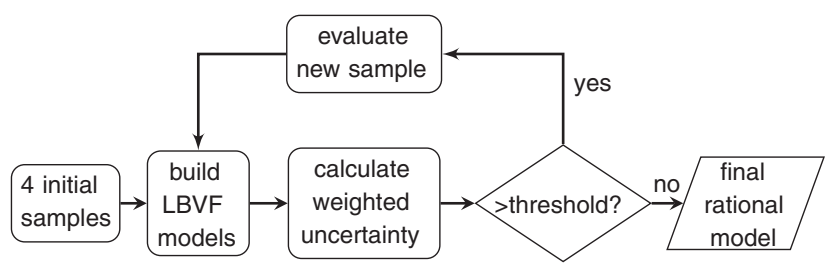

Fig. 1 Flowchart of proposed AFS strategy

A pronounced advantage of the proposed scheme over classical AFS schemes [1-4] is its capacity to sample not only where models of a different order disagree, but also where they may agree in the mean, but show a large variance. As such, this results in a more careful stopping criterion. The cost of the advantages is that for every sample of $\widehat{r_{k}}$ and $\widehat{d}$ that is drawn, an eigenvalue problem must be solved and a QR decomposition performed to find the corresponding poles and residues, though the computational cost involved is usually negligible with respect to the EM-simulation cost. 

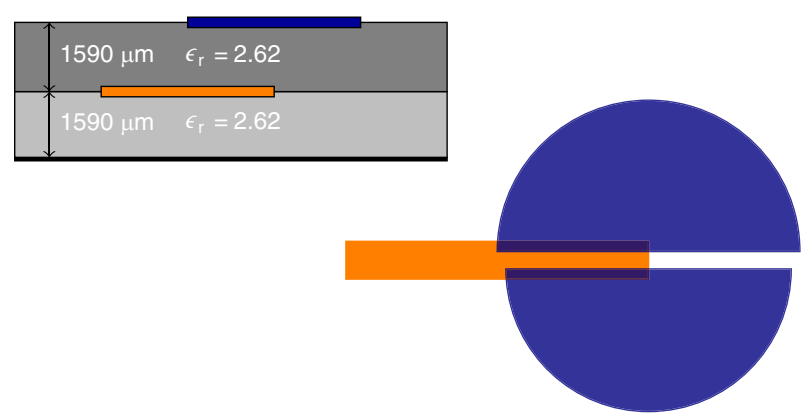

Fig. 2 Design of semi-circular patch antenna. Two semi-circular patches of different radii (17.5 and 16.5, and $2 \mathrm{~mm}$ apart) are indirectly excited by a microstrip line of width $4.373 \mathrm{~mm}$

Example: The proposed method is applied to the double semi-circular patch antenna $[2,7]$ shown in Fig. 2.

An example of the results obtained in one iteration of the proposed Bayesian AFS scheme is shown in Fig. 3. The black points represent the known simulated points (where EM simulations have been performed). A thousand samples from the LBVF models with four, five, and six poles are plotted in three shades of purple, orange, and red according to the likelihood of the corresponding model. Above this plot, in green, the uncertainty measure is shown (though shifted and rescaled to be discernible above the rest of the figure). The green arrow underneath indicates the maximum of the uncertainty, and thus where the next frequency point will be chosen.

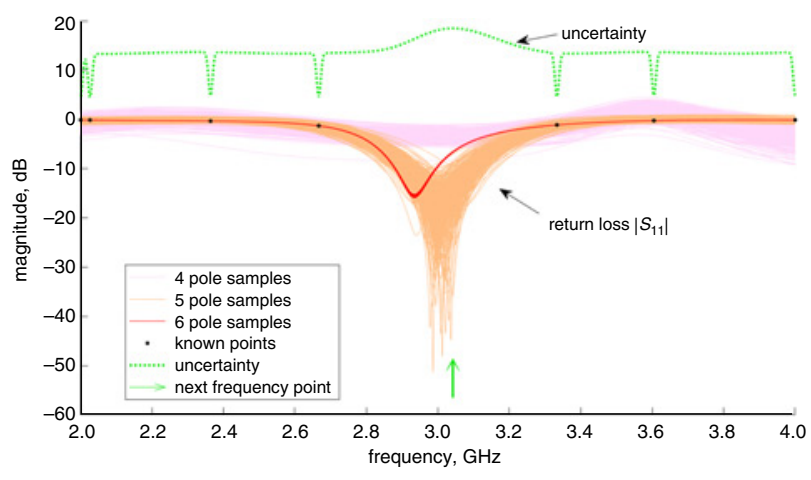

Fig. 3 Step in adaptive sampling scheme. Uncertainty does not conform to vertical axis, but is rescaled and shifted for clarity

Fig. 4 displays the marginal log-likelihood of the pole relocation system at this step. This does not necessarily increase monotonically with the number of poles, as is the case in this intermediate stage.

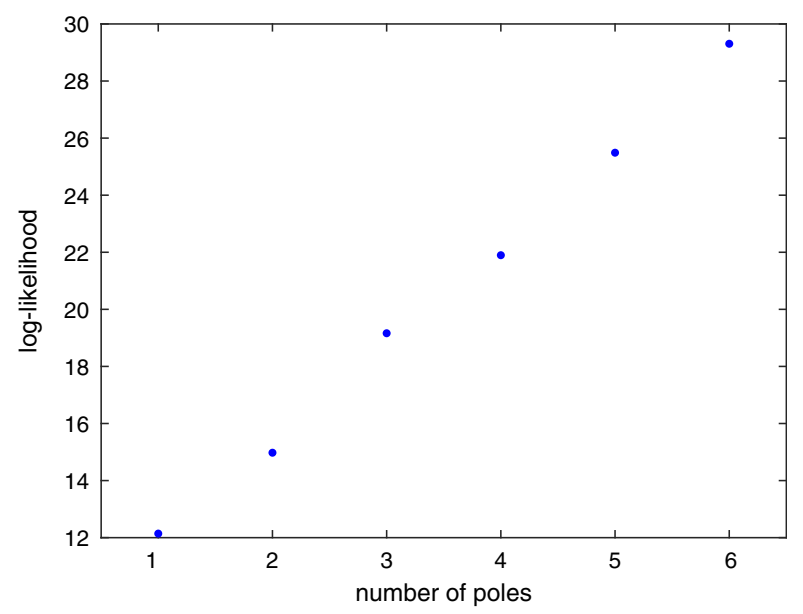

Fig. 4 Marginal log-likelihood of data for pole relocation system as function of number of poles used in each model, at same stage as Fig. 3

In this example, a threshold of $-80 \mathrm{~dB}$ has been chosen for the uncertainty measure. This criterion is satisfied after 11 EM simulations and the final (mean) fit is shown in Fig. 5.
The root-mean-square (rms) and maximum error with respect to the sampled frequency points are -138.3 and $-130.9 \mathrm{~dB}$, respectively. With respect to the antenna response calculated for 10,000 frequency points in the range of $2-4 \mathrm{GHz}$, the rms and maximum error are -82.1 and $-72.8 \mathrm{~dB}$, respectively. For comparison, the ad hoc method described in [2] reports a fitting error of $<-70 \mathrm{~dB}$ for the same example, also for $11 \mathrm{EM}$ simulations.

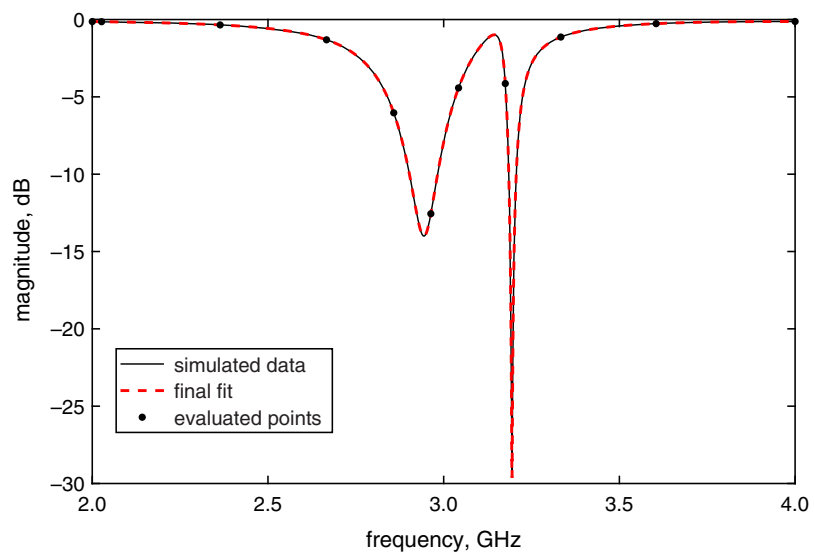

Fig. 5 Best fit (with ten poles) after reaching threshold

Conclusion: This Letter introduces a novel AFS method, based on a Bayesian treatment of the well-established VF method. The method makes use of samples from the posterior distribution of poles and residues to construct a probabilistic uncertainty measure. For this, it automatically weighs models of different orders by their marginal likelihood. This uncertainty measure is then used to iteratively select, in a principled way, new frequencies where additional EM simulations have to be performed.

The method is applied to an asymmetric double semi-circular patch antenna and is shown to efficiently reach an accurate fit to the simulated data, proving its efficacy.

(C) The Institution of Engineering and Technology 2019 Submitted: 19 August 2018 E-first: 26 November 2018 doi: $10.1049 / \mathrm{el} .2018 .6668$

One or more of the Figures in this Letter are available in colour online. S. De Ridder, D. Deschrijver, D. Spina, T. Dhaene and D. Vande Ginste (IDLab, Department of Information Technology, Ghent Universityimec, 9000 Gent, Belgium)

凶-mail: simon.deridder@ugent.be

\section{References}

1 Antonini, G., Deschrijver, D., and Dhaene, T.: 'Broadband rational macromodeling based on the adaptive frequency sampling algorithm and the partial element equivalent circuit method', Trans. Electromagn. Compat., 2008, 50, pp. 128-137

2 Dhaene, T., Ureel, J., Faché, N., et al.: 'Adaptive frequency sampling algorithm for fast and accurate S-parameter modeling of general planar structures'. Proc. 1995 IEEE MTT-S Int. Microwave Symp., Orlando, FL, USA, May 1995, vol. 3, pp. 1427-1430

3 Deschrijver, D., and Dhaene, T.: 'Passivity-based sample selection and adaptive vector fitting algorithm for pole-residue modeling of sparse frequency-domain data'. Proc. IEEE Behavioral Modeling and Simulation (BMAS), San Jose, CA, USA, October 2004, pp. 68-73

4 Mutonkole, N., and de Villiers, D.I.L.: 'Adaptive frequency sampling for radiation patterns and S-parameters of antennas'. Proc. 11th European Conf. on Antennas and Propagation (EUCAP), Paris, France, March 2017, pp. 3195-3199

5 Gustavsen, B., and Semlyen, A.: 'Rational approximation of frequency domain responses by vector fitting', Trans. Power Deliv., 1999, 14, pp. $1052-1061$

6 Gustavsen, B.: 'Improving the pole relocating properties of vector fitting', Trans. Power Deliv., 2006, 21, pp. 1587-1592

7 Alonso-Monferrer, F., Kishk, A.A., and Glisson, A.W.: 'Green's functions analysis of planar circuits in a two-layer grounded medium', Trans. Antennas Propag., 1992, 40, pp. 690-696 